Review Article

\title{
Psoriasis and Risk of Uveitis: A Systematic Review and Meta- Analysis
}

\author{
Chayada Chaiyabutr $\mathbb{D}^{1},{ }^{1}$ Patompong Ungprasert, ${ }^{2}$ Narumol Silpa-archa, ${ }^{1}$ \\ Chanisada Wongpraparut, ${ }^{1}$ and Leena Chularojanamontri ${ }^{1}{ }^{1}$ \\ ${ }^{1}$ Department of Dermatology, Faculty of Medicine Siriraj Hospital, Mahidol University, Bangkok, Thailand \\ ${ }^{2}$ Clinical Epidemiology Unit, Department of Research and Development, Faculty of Medicine Siriraj Hospital, Mahidol University, \\ Bangkok, Thailand
}

Correspondence should be addressed to Leena Chularojanamontri; leenajim@gmail.com

Received 20 April 2020; Revised 28 May 2020; Accepted 27 June 2020; Published 16 July 2020

Academic Editor: Vinod Chandran

Copyright (c) 2020 Chayada Chaiyabutr et al. This is an open access article distributed under the Creative Commons Attribution License, which permits unrestricted use, distribution, and reproduction in any medium, provided the original work is properly cited.

\begin{abstract}
Background. Uveitis is a known ophthalmologic manifestation of seronegative spondyloarthropathy, including psoriatic arthritis. However, the data is less clear among patients with psoriasis due to the limited number of published studies. Aims. To investigate whether the risk of incident and prevalent uveitis is elevated among patients with psoriasis using systematic review and meta-analysis technique. Methods. The MEDLINE and EMBASE databases were searched from their inception to May 2019. Eligible studies must have included a psoriasis group and a nonpsoriasis group. Eligible studies must also have investigated for prevalent or incident uveitis, and the magnitude of difference between the study groups must have been reported. Pooled risk ratio and 95\% confidence interval (CI) were calculated using random-effect generic inverse variance methods. Results. Of 7,107 potentially eligible articles from the EMBASE and MEDLINE databases, 7 studies were included in the meta-analysis. Two of those studies compared the incidence, and 5 studies compared the prevalence of uveitis between the psoriasis and nonpsoriasis groups. For incident uveitis, a total of 5,865,801 patients $(222,083$ with psoriasis and 5,643,718 without psoriasis) were analyzed. For prevalent uveitis, a total of 1,343,436 patients $(37,891$ with psoriasis and 1,305,545 without psoriasis) were studied. The risk of incident uveitis was significantly higher among patients with psoriasis with a pooled risk ratio of 1.23 (95\% CI: $\left.1.05-1.45, I^{2}=55 \%\right)$. The risk of prevalent uveitis was also significantly higher among patients with psoriasis with a pooled risk ratio of 1.97 (95\% CI: $\left.1.68-2.31, I^{2}=0 \%\right)$. Conclusions. The results of this study revealed significantly increased risk of both prevalent and incident uveitis among patients with psoriasis.
\end{abstract}

\section{Introduction}

Psoriasis is a common chronic inflammatory skin disease with a reported prevalence that ranges from $0.5 \%$ to $11.4 \%$ depending on geographic region [1]. Its pathogenesis is related to autoimmunity and systemic inflammation, which can result in multiorgan involvement and increased risk of comorbidities. Ocular involvement is being increasingly recognized as a complication of psoriasis [2-4]. The possible mechanisms of ocular involvement include direct involvement of psoriatic skin lesion to the epithelial component of the eye, shared genetic predisposition, and adverse effect of psoriasis treatment, such as excessive use of topical corti- costeroids and prolonged course of psoralen-UVA (PUVA) photochemotherapy [2].

Uveitis is characterized by inflammation of the uveal tract, which includes the iris, ciliary body, choroid tissue, and adjacent structures [5]. The reported global prevalence of uveitis ranged from 38 to 714 cases per 100,000 individuals. If left untreated, uveitis can lead to several complications that can lead to permanent visual impairment [6]. Uveitis is a known ophthalmologic manifestation of several seronegative spondyloarthropathies, such as ankylosing spondylitis, reactive arthritis, and psoriatic arthritis. It is also being increasingly reported in psoriasis [7]. However, the results of studies of uveitis in psoriasis are fairly heterogeneous, and some studies 


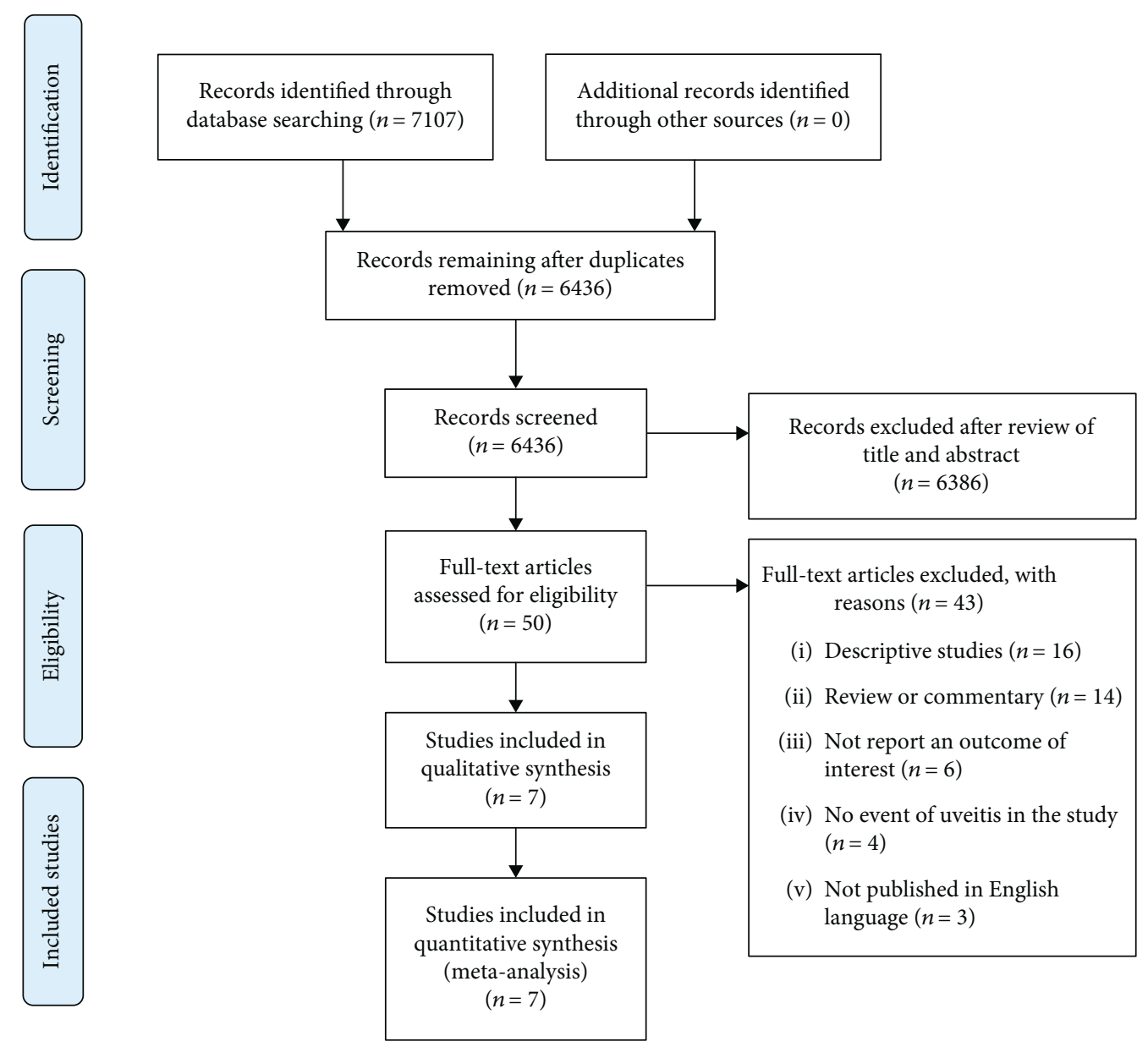

Figure 1: Flow chart describing the literature review and selection process.

are just descriptive studies without comparative analysis [3, 8-11]. The aim of this study was to better understand and characterize the association between psoriasis and uveitis by identifying all related studies and systematically summarizing their results.

\section{Materials and Methods}

2.1. Search Strategy. Two investigators (C.C. and L.C.) independently searched published studies indexed in the MEDLINE and EMBASE databases from inception to May 2019. The search strategy included terms for "psoriasis" and "eye disease," as described in Table S1. Eligible studies must include (i) a cohort of patient with adult psoriasis (cases) and a cohort of patients without psoriasis (comparators) and (ii) reported the difference of prevalent or incident uveitis between the two study groups. The reported difference could be in the form of odds ratio (OR), relative risk (RR), hazard ratio (HR), or standardized incidence ratio (SI) with 95\% confidence interval (CI). Alternatively, if those ratios were not reported, sufficient raw data to calculate them was considered acceptable. Non-full-text articles were excluded.

The title and abstract of the retrieved articles were independently reviewed by two investigators (C.C. and L.C.).
Articles that obviously did not fulfill the inclusion criteria were screened out during this round. Full-text articles among the remaining potentially eligible articles were then independently reviewed by the same two investigators for final determination of their inclusion eligibility. Any disagreement in the determination of reviewed studies was resolved by discussion and consensus between the two investigators. Quality assessment of the included studies was performed using the NewcastleOttawa quality assessment scale. This tool is specifically designed for assessment of quality of nonrandomized study, such as case-control and cohort study. It assesses the quality based on three aspects, including the selection of case and control, the comparability between the groups, and the ascertainment of the outcome of interest for cohort study and exposure of interest for case-control study [12]. Study with score $\geq 7$ is generally considered as a high-quality study [13].

2.2. Data Extraction. The following data were extracted using a standardized data collection form: first author's name, title of the study, journal name, year of publication, year when the study was conducted, country where the study was conducted, study design, method used to diagnose psoriasis and uveitis, recruitment of cases and comparators, number and baseline characteristics of cases and comparators, follow-up duration, variables that were adjusted in multivariate analysis, and 
TABLE 1: Characteristics of included incident studies.

\begin{tabular}{lcc}
\hline & Egeberg et al. [17] & Chi et al. [16] \\
\hline Country of origin & Denmark & Taiwan \\
Study design & Cohort study & Cohort study \\
Year of publication & 2015 & 2017
\end{tabular}

Cases of adults (18 years or older) with psoriasis were identified from the Danish National Patient Registry during 1 January 1997

to 31 December 2011. This registry covers all citizens of Denmark.

Diagnosis of psoriasis was made based on the presence of Cases

Comparators

Diagnosis of incident uveitis

The presence of the first diagnosis code for uveitis in the database after the index date.

$74,129 / 5,434,749$

$50.6 / 51.6$

$40.7 / 43.3$

Until 31 December 2011, a diagnosis of end point, migration, or death from any causes.

Confounder assessed in the multivariate analysis

Quality assessment diagnostic codes of psoriasis (ICD-8 code of 696.10 or 696.19 or -10 code of L40, which are codes for psoriasis in general) or derivatives in the absence of ICD- 8 code of 696.09 or ICD-10 included.

Comparators were the rest of patients in the database who did not carry the diagnostic codes of psoriasis/psoriatic arthritis.

Comparators with diagnosis of uveitis prior to index date were not included.

Number of subjects

Percentage of female gender

Mean age in years

(cases/comparators)

Follow-up

Age, gender, socioeconomic status, and comorbidities

Selection: 4 stars

Comparability: 1 star

Outcome: 3 stars
Cases of psoriasis were identified from the National Health Insurance Research Database during 2000 to 2011 . This registry covers $99 \%$ of the citizens of Taiwan.

Diagnosis of psoriasis was made based on the presence of diagnostic codes of psoriasis (ICD9-CM code of 696, 696.1, or 696.8, which are codes for psoriasis in general), in the absence of ICD-9-CM code of 696.0, which is the code for psoriatic arthritis.

Cases with diagnosis of uveitis prior to index date were not included.

Gender- and age-matched comparators without a diagnosis code for psoriasis were randomly selected from the 2005 Longitudinal Health Insurance Database, which is a random subset of one million enrollees in the National Health Insurance Research Database for the year 2005. Comparators with diagnosis of uveitis prior to index date were not included.

The presence of the first diagnosis code for uveitis in the database after the index date (at least twice for outpatient visit or once for hospital admission).

$147,954 / 147,954$

$41.2 / 41.2$

$44.4 / 44.4$

Until incident uveitis, 31 December 2012, or withdrawal from the National Health Insurance program.

Age, gender, hypertension, dyslipidaemia, and diabetes

Selection: 4 stars

Comparability: 2 stars

Outcome: 3 stars adjusted effect estimates with corresponding 95\% CIs. Data extraction was independently performed by C.C. and L.C. to minimize any errors.

2.3. Statistical Analysis. Statistical analysis was performed using Review Manager 5.3 software from the Cochrane Collaboration (London, UK). Result of each study was combined to calculate the pooled effect using the generic inverse variance method of DerSimonian and Laird [14]. This method gives higher weight for the pooled analysis to study with higher precision. The weight for each study is in reverse to its variance as study with higher variance is study with less precision. The random-effect model was used in this analysis because the assumption of fixed-effect model that every study should give rise to the same result is generally not true, particularly in observational studies. Cochran's $Q$ test and $I$ ${ }^{2}$ statistic were used to assess for between-study statistical heterogeneity [15]. This $I^{2}$ statistic was used to quantify the proportion of the total variation across studies that was from heterogeneity rather than chance. A value of $I^{2}$ of $0-25 \%$ represents insignificant heterogeneity, 26-50\% low heterogeneity, $51-75 \%$ moderate heterogeneity, and $>75 \%$ high heterogeneity. Visualization by funnel plot was used for determination of publication bias if there were enough eligible studies to create the plot. Publication bias would be suspected if the funnel plot is asymmetric. 
TABLE 2: Characteristics of included prevalent studies.

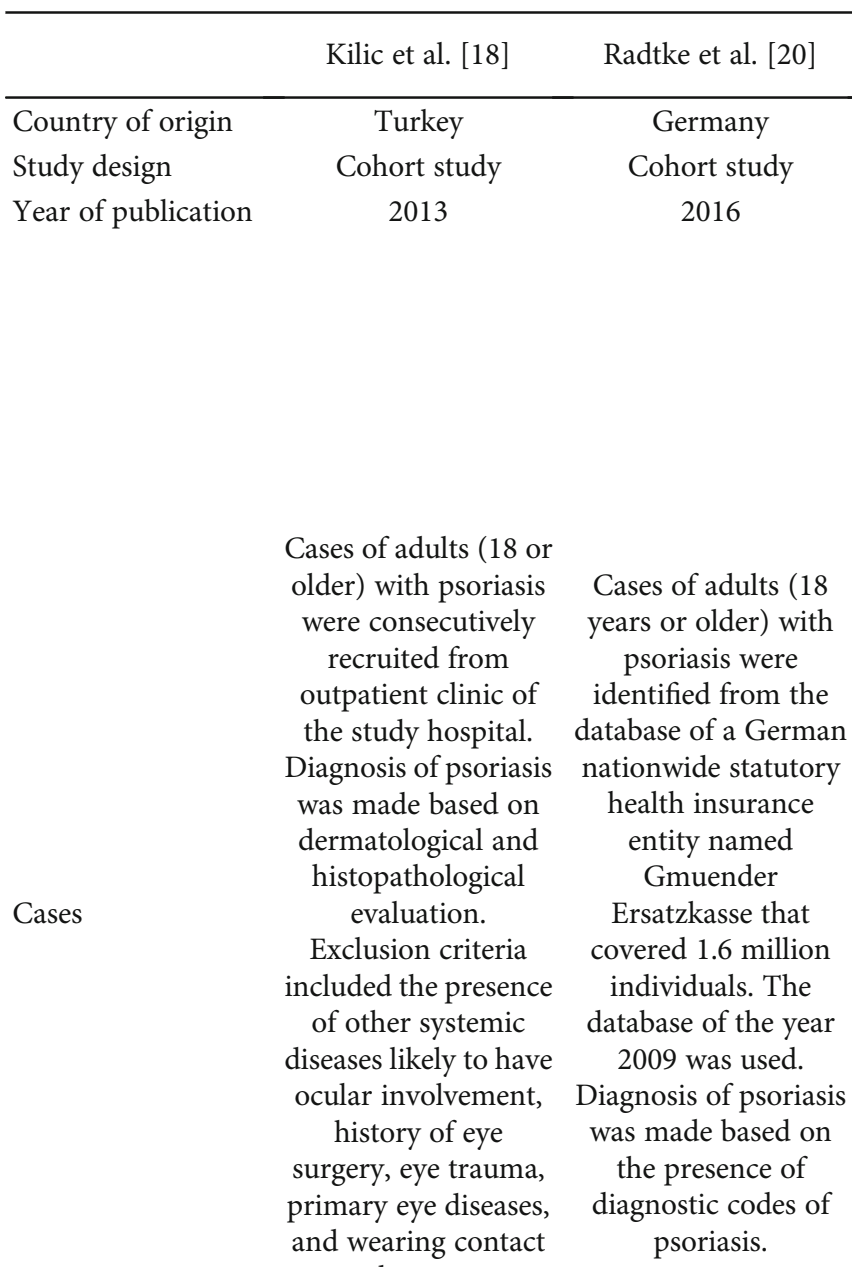
lenses.

Comparators

Comparators without psoriasis and with healthy first-degree relatives were consecutively recruited from the same center.

Diagnosis of
prevalent uveit

Number of subjects (cases/comparators) ophthalmologists.
Comparators were the rest of patients in the database who did not carry the diagnostic codes of psoriasis

The presence of the first diagnosis code for uveitis in the database.

$$
\begin{aligned}
& 100 / 100 \\
& 52.0 / 52.0
\end{aligned}
$$

Cases with psoriasis

who were followed by the dermatology clinic of the study enrolled.

Exclusion criteria included prior ocular surgery, retinal or choroidal pathology, high myopia and

hypermetropia, bestcorrected visual acuity less than 20/25, systemic disease (hypertension, diabetes mellitus, cerebrovascular disease, peripheral vascular disease, pregnancy, or lactation), and history of systemic treatment for psoriasis. hospital were
Cases with psoriasis the outpatient clinic at the Faculty of Medicine, Alexandria University,

Alexandria, Egypt

Exclusion criteria included inflammatory connective tissue diseases, diabetes, nephropathy, lung and heart disease, gastroenterological disease/inflammatory bowel disease, neurological disease, neoplasia, metabolic bone disease, skin diseases other than psoriasis, infections, hematological disease, liver disease, previous ocular surgery, active eye infection, or active ocular allergy. were recruited from
Comparators were individuals without psoriasis who were admitted to the outpatient Clinic of Ophthalmology for minor refractive errors. They were gender- and agematched to cases.

Comparators were individuals without psoriasis who were seen at the same clinic. They were gender- and agematched to cases.
Ghalamkarpour et al. [4]

$$
\begin{gathered}
\text { Cohort study } \\
2019
\end{gathered}
$$

Cases of adults (18 years or older) with psoriasis were recruited from two dermatology centers from September 2014 to January 2017. Exclusion criteria included diabetes mellitus, hypertension, hyperlipidemia, thyroid disorders, hepatic or renal insufficiency,

rheumatoid arthritis, gout, collagen vascular diseases, atopic dermatitis, rosacea, other chronic inflammatory

diseases, pregnant or lactating women, smokers, contact lens wearers, use of anticholinergics,

history of eye trauma or surgery, optic neuritis, glaucoma, radiotherapy, malignancy or chronic infection of the lacrimal glands, human

immunodeficiency

virus, and hepatitis $B$ or $\mathrm{C}$ virus infections.

Based on examination Based on examination Based on examination by ophthalmologists. by ophthalmologists. by ophthalmologists.
parators without same centers.


TABle 2: Continued.

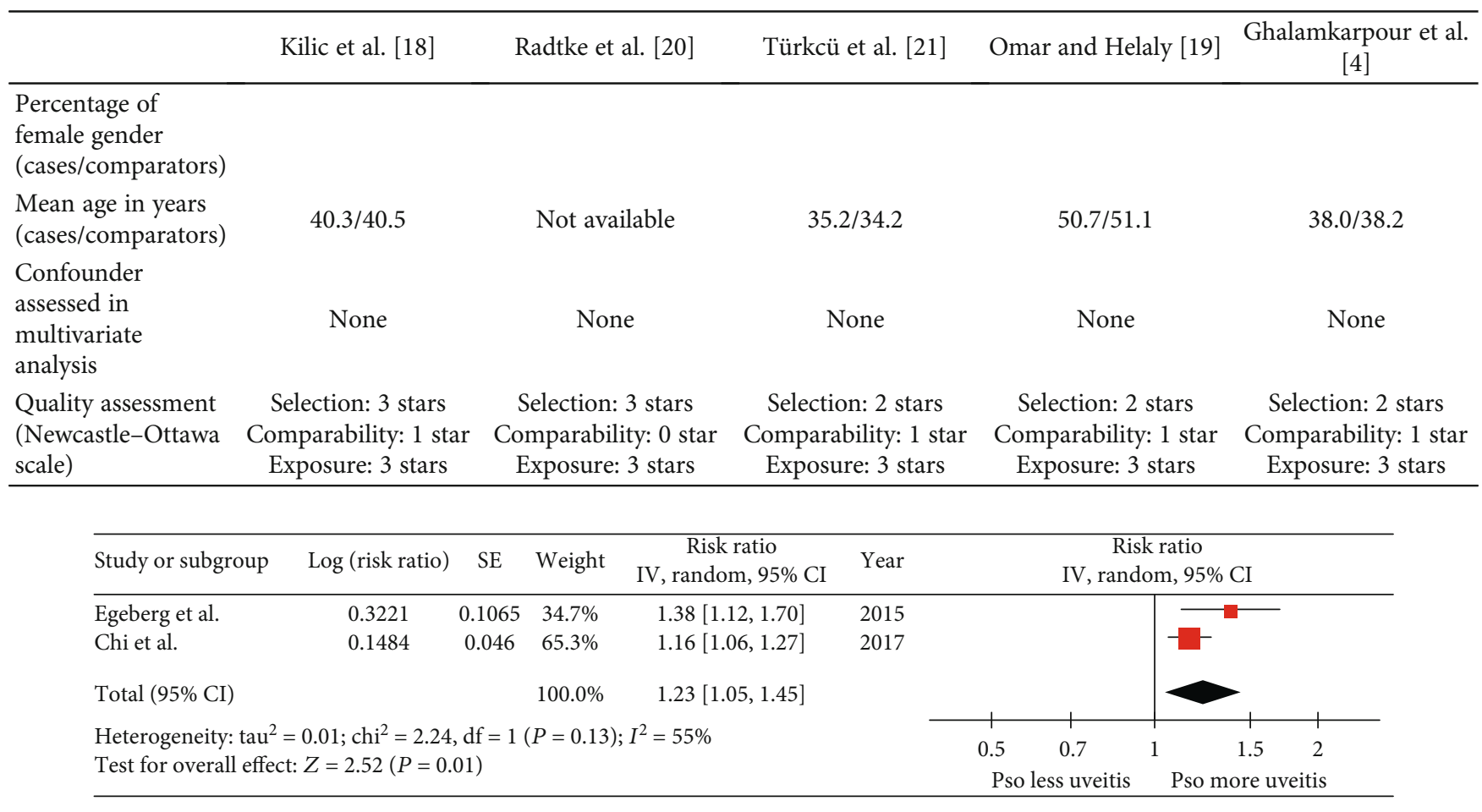

FIGURE 2: Forest plot of risk of incident uveitis.

\begin{tabular}{|c|c|c|c|c|c|c|c|c|c|c|c|}
\hline Study or subgroup & Log (risk ratio) & SE & Weight & $\begin{array}{c}\text { Risk ratio } \\
\text { IV, random, 95\% CI }\end{array}$ & Year & \multicolumn{6}{|c|}{$\begin{array}{c}\text { Risk ratio } \\
\text { IV, random, 95\% CI }\end{array}$} \\
\hline Kilic et al. & 1.6094 & 1.5428 & $0.3 \%$ & $5.00[0.24,102.85]$ & 2013 & & & & & & \\
\hline Turkcu et al. & 2.2484 & 1.4566 & $0.3 \%$ & $9.47[0.55,164.56]$ & 2016 & & & & & & \\
\hline Radtke et al. & 0.6678 & 0.0822 & $98.9 \%$ & $1.95[1.66,2.29]$ & 2017 & & & & & & \\
\hline Omar et al. & 1.9459 & 1.5053 & $0.3 \%$ & $7.00[0.37,133.78]$ & 2018 & & & & & & \\
\hline Ghalamkarpour et al. & 0.4104 & 1.6284 & $0.3 \%$ & $1.51[0.06,36.67]$ & \multirow[t]{2}{*}{2019} & & & & & & \\
\hline Total $(95 \%$ CI $)$ & & & $100.0 \%$ & $1.97[1.68,2.31]$ & & & & & & & \\
\hline \multirow{2}{*}{\multicolumn{6}{|c|}{$\begin{array}{l}\text { Heterogeneity: } \operatorname{tau}^{2}=0.00 ; \mathrm{chi}^{2}=2.28, \mathrm{df}=4(P=0.68) ; I^{2}=0 \% \\
\text { Test for overall effect: } Z=8.30(P<0.00001)\end{array}$}} & $0.1 \quad 0.2$ & 0.5 & 1 & 2 & 5 & 10 \\
\hline & & & & & & \multicolumn{2}{|c|}{ Pso less uveitis } & \multicolumn{4}{|c|}{ Pso more uveitis } \\
\hline
\end{tabular}

FIGURE 3: Forest plot of risk of prevalent uveitis.

\section{Results}

The search strategy identified 7,107 potentially eligible articles from the EMBASE and MEDLINE databases. After exclusion of 671 duplicate articles, the titles and abstracts of the remaining 6,436 articles were reviewed. That review yielded 50 studies for full-text review. Forty-three of those studies were excluded for the following reasons: descriptive study $(n=16)$, review or commentary $(n=14)$, did not report an outcome of interest $(n=6)$, no event of uveitis in the study $(n=4)$, and not published in English language $(n=3)$. The remaining 7 seven studies were included in the meta-analysis.

Of those, 2 studies $[16,17]$ compared the incidence, and 5 studies [4, 18-21] compared the prevalence of uveitis between the psoriasis and nonpsoriasis groups. A flowchart describing the literature review and selection process is shown in Figure 1. The detail of psoriasis and nonpsoriasis group from each study, characteristics of the included inci- dence, and prevalence studies and quality assessment are shown in Tables 1 and 2, respectively.

The incidence of uveitis ranged from 2.9 per 10,000 person-years in the study by Egeberg et al. [17] to 10.4 per 10,000 person-years in the study by Chi et al. [16]. The prevalence of uveitis ranged from $0.4 \%$ in the study by Radtke et al. [20] to $14.3 \%$ in the study by Türkcü et al. [21].

3.1. Risk of Incident Uveitis. A total of 222,083 patients with psoriasis and 5,643,718 individuals without psoriasis were included in the incident uveitis analysis. The risk of incident uveitis was significantly higher among patients with psoriasis than among those without psoriasis with a pooled risk ratio of 1.23 (95\% CI: 1.05-1.45). The statistical heterogeneity was moderate $\left(I^{2}=55 \%\right)$ (Figure 2$)$. The quality of both studies was good. Evaluation for publication bias using funnel plot was not performed due to the small number of included studies. 


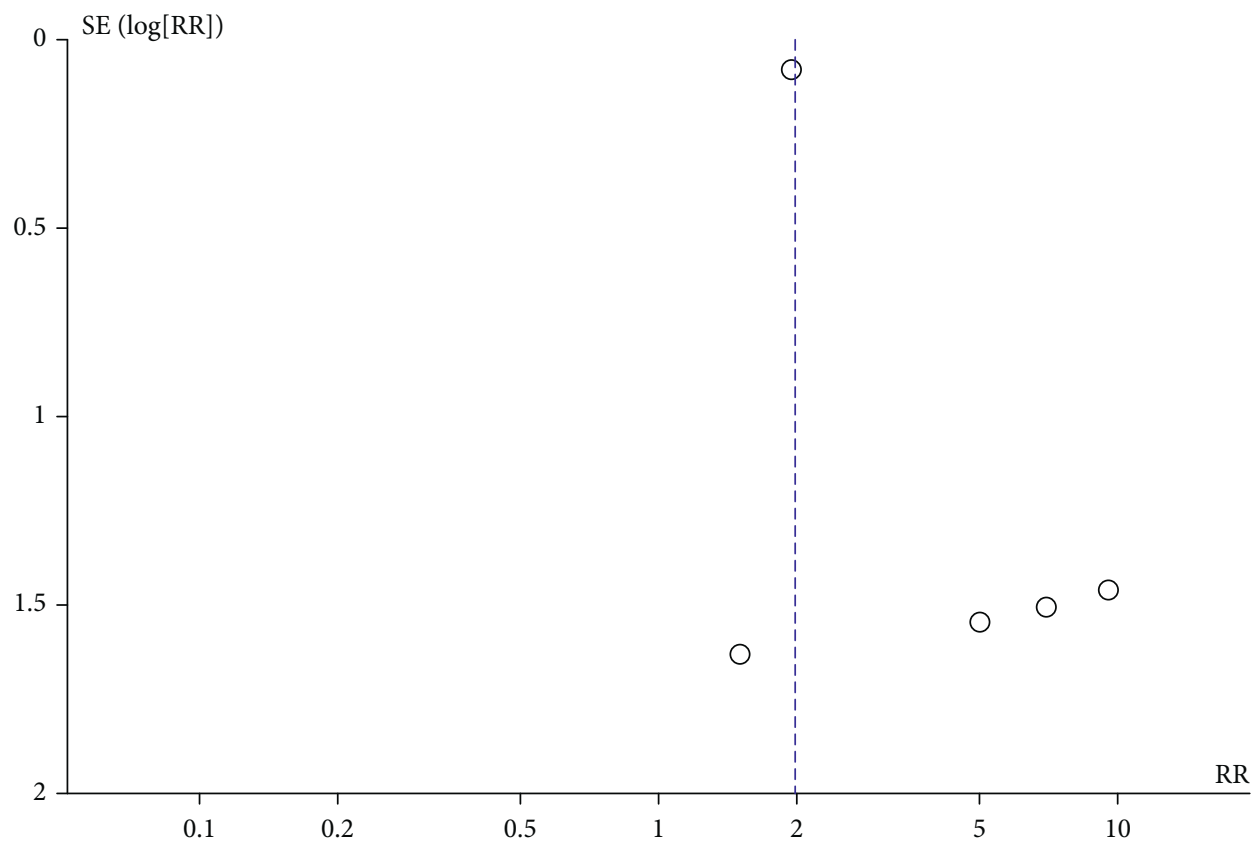

Figure 4: Funnel plot of prevalent uveitis.

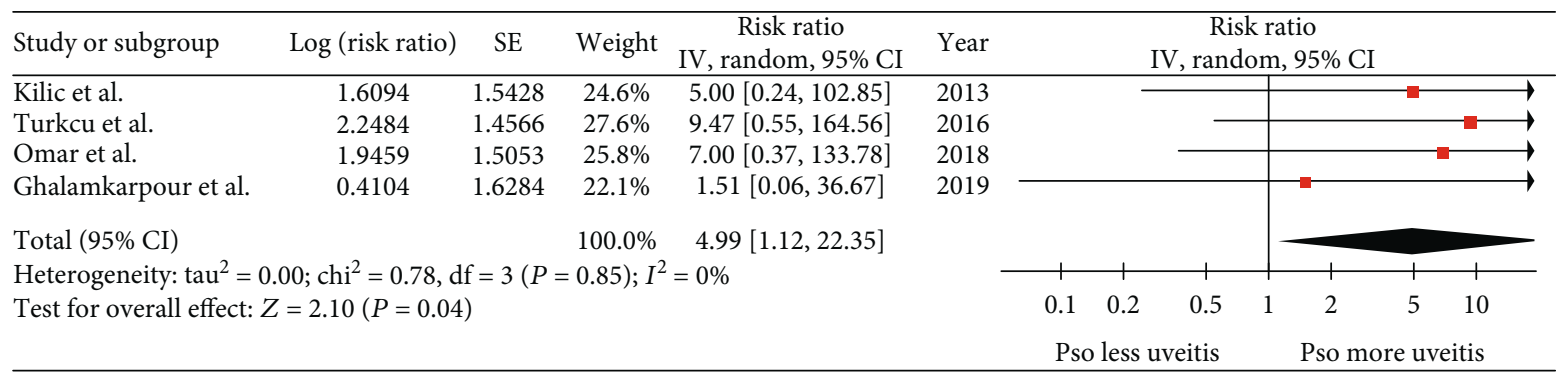

FIGURE 5: Sensitivity analysis of risk of prevalent uveitis.

3.2. Risk of Prevalent Uveitis. A total of 37,891 patients with psoriasis and 1,305,545 individuals without psoriasis were included in the prevalent uveitis analysis. The risk of prevalent uveitis was also significantly higher among patients with psoriasis than among nonpsoriasis subjects with a pooled risk ratio of 1.97 (95\% CI: 1.68-2.31). The quality of the included studies was fair to good. The heterogeneity analysis revealed no statistical heterogeneity $\left(I^{2}=0 \%\right)$ (Figure 3 ). Funnel plot was relatively symmetric and was not suggestive of the presence of publication bias (Figure 4).

Sensitivity analysis was conducted to explore whether the pooled result would be significantly different if the dominant study was not included. After excluding the study by Radtke et al. [20], we found that the risk of prevalent uveitis remained significantly higher among patients with psoriasis than individuals without psoriasis. The new pooled risk ratio was increased to 4.99 (95\% CI: $1.12-22.35 ; I^{2}=0 \%$ ) (Figure 5).

\section{Discussion}

Uveitis is a known ophthalmologic manifestation of several systemic diseases, including seronegative spondyloarthropathy [7]. Acute unilateral anterior uveitis is the classic and well-recognized inflammatory eye disease in patients with ankylosing spondylitis, but all of the diseases in the seronegative spondyloarthropathy group could manifest with uveitis, including psoriatic arthritis [22]. However, the data is less clear among patients with psoriasis due to the limited number of studies addressing this issue. Accordingly, the present systematic review and meta-analysis combined all of the available data and found both incident and prevalent uveitis to be significantly more common among patients with psoriasis than among general population. For a sensitivity analysis, the study by Radtke et al. [20] was excluded from the full analysis because this study received almost $99 \%$ of weight in the pooled analysis as it was conducted using a very large administrative database. Interestingly, even after excluding this study, the pooled risk ratio was increased from 1.97 to 4.99. This finding supports that patients with psoriasis have a higher risk of prevalent uveitis than individuals without psoriasis.

There are few possible explanations for the observed increased risk of uveitis among psoriasis patients. The first explanation is related to the increased systematic inflammatory burden among patients with psoriasis that may also trigger ocular inflammation. In fact, several cytokines that 
are known to be key players in the pathogenesis of psoriasis, such as tumor necrosis factor- (TNF-) alpha, interleukin(IL-) 2, IL-6, and IL-17, are also found to be at an increased concentration in the aqueous humor of patients with uveitis $[23,24]$. Second, both diseases may share some genetic predisposition that could ultimately lead to autoimmunity in both the eye and the skin. One widely known example is HLA-B27, which is known to be associated with both psoriatic arthritis and uveitis $[25,26]$.

There are case reports of patients with psoriasis and other immune-mediated inflammatory diseases who developed uveitis after exposure to TNF-alpha inhibitor [27]. It is possible that they are true cases of TNF-alpha inhibitor-induced uveitis, and thus, use of TNF-alpha inhibitor could be one of the explanations for the increased prevalence/incidence of uveitis among patients with psoriasis. However, it is more likely that those patients developed uveitis because of their underlying disease (such as seronegative spondyloarthropathies) rather than as a complication of TNF-alpha inhibitor since TNF-alpha inhibitor has been extensively shown to be beneficial for mostly all types of uveitis [28].

\section{Limitations}

This study has some limitations that should be acknowledged. First, most included studies relied on diagnostic codes to make the diagnosis of psoriasis and uveitis, which may have limited the diagnostic accuracy for both conditions. This is of particular concern for uveitis as other inflammatory orbital diseases may be erroneously coded as uveitis. Additionally, traumatic anterior uveitis, which is not an autoimmune-related uveitis, could be coded under the same diagnostic codes for uveitis. This also limits the ability to further characterize subtype of uveitis. Second, moderate heterogeneity was observed in the meta-analysis of incident uveitis, which suggests that the included studies may have been somewhat too different to combine. Third, the quality of some of the included studies was low as reflected by the low Newcastle-Ottawa scores. The last point is that some of psoriasis patients in our cohort may have psoriasis in conjunction with psoriatic arthritis. Therefore, this result may not totally reflect the risk of uveitis in patients who have only skin psoriasis.

\section{Conclusion}

The results of this study revealed significantly increased risk of both prevalent and incident uveitis among patients with psoriasis. Dermatologists should regularly monitor these patients for eye symptoms for prompt recognition and treatment to prevent irreversible ocular complications.

\section{Conflicts of Interest}

All authors declare no personal or professional conflicts of interest relating to any aspect of this study.

\section{Authors' Contributions}

Patompong Ungprasert and Leena Chularojanamontri contributed equally to this article.

\section{Supplementary Materials}

Table S1: database search strategy. (Supplementary Materials)

\section{References}

[1] I. M. Michalek, B. Loring, and S. M. John, "A systematic review of worldwide epidemiology of psoriasis," Journal of the European Academy of Dermatology and Venereology, vol. 31, no. 2, pp. 205-212, 2017.

[2] B. Rehal, B. S. Modjtahedi, L. S. Morse, I. R. Schwab, and H. I. Maibach, "Ocular psoriasis," Journal of the American Academy of Dermatology, vol. 65, no. 6, pp. 1202-1212, 2011.

[3] N. S. Chandran, M. Greaves, F. Gao, L. Lim, and B. C. L. Cheng, "Psoriasis and the eye: prevalence of eye disease in Singaporean Asian patients with psoriasis," The Journal of Dermatology, vol. 34, no. 12, pp. 805-810, 2007.

[4] F. Ghalamkarpour, A. Baradaran-Rafii, M. M. Sadoughi et al., "Ocular findings in patients with psoriasis: is it related to the side effects of treatment or to psoriasis itself? A case-control study," The Journal of Dermatological Treatment, vol. 31, no. 1, pp. 27-32, 2020.

[5] T. Barisani-Asenbauer, S. M. Maca, L. Mejdoubi, W. Emminger, K. Machold, and H. Auer, "Uveitis- a rare disease often associated with systemic diseases and infections- a systematic review of 2619 patients," Orphanet Journal of Rare Diseases, vol. 7, no. 1, p. 57, 2012.

[6] T. Tsirouki, A. Dastiridou, C. Symeonidis et al., "A focus on the epidemiology of uveitis," Ocular Immunology and Inflammation, vol. 26, no. 1, pp. 2-16, 2016.

[7] S. M. Sharma and D. Jackson, "Uveitis in the Spondyloarthopathies," Best Practice \& Research Clinical Rheumatology, vol. 31, no. 6, pp. 846-862, 2017.

[8] D. L. Knox, "Psoriasis and intraocular inflammation," Transactions of the American Ophthalmological Society, vol. 77, pp. 210-224, 1979.

[9] M. D. Riveira and N. A. Alba, "UVEITIS in psoriasic patients: a descriptive study," Investigative Ophthalmology \& Visual Science, vol. 56, p. 1865, 2015.

[10] R. Tanaka, M. Takamoto, K. Komae, K. Ohtomo, Y. Fujino, and T. Kaburaki, "Clinical features of psoriatic uveitis in Japanese patients," Graefe's Archive for Clinical and Experimental Ophthalmology, vol. 253, no. 7, pp. 1175-1180, 2015.

[11] P. Yang, M. Zheng, L. Zhang et al., "Uveitis in Chinese patients with psoriasis," Ocular Immunology and Inflammation, vol. 25, pp. 855-865, 2016.

[12] G. Wells, B. Shea, D. O'Connell, J. Peterson, V. Welch, M. Losos et al., The Newcastle-Ottawa Scale (NOS) for assessing the quality of nonrandomised studies in meta-analyses, 2000, http://www.ohri.ca/programs/clinical_epidemiology/ oxford.asp.

[13] J. K. Harrison, J. Reid, T. J. Quinn, and S. D. Shenkin, "Using quality assessment tools to critically appraise ageing research: a guide for clinicians," Age and Ageing, vol. 46, no. 3, pp. 359-365, 2017. 
[14] R. DerSimonian and N. Laird, "Meta-analysis in clinical trials," Controlled Clinical Trials, vol. 7, no. 3, pp. 177-188, 1986.

[15] J. P. Higgins, S. G. Thompson, J. J. Deeks, and D. G. Altman, "Measuring inconsistency in meta-analyses," BMJ, vol. 327, no. 7414, pp. 557-560, 2003.

[16] C. C. Chi, T. H. Tung, J. Wang et al., "Risk of uveitis among people with psoriasis: a nationwide cohort study," JAMA Ophthalmology, vol. 135, no. 5, pp. 415-422, 2017.

[17] A. Egeberg, U. Khalid, G. H. Gislason, L. Mallbris, L. Skov, and P. R. Hansen, "Association of psoriatic disease with uveitis: a Danish nationwide cohort study," JAMA Dermatology, vol. 151, no. 11, pp. 1200-1205, 2015.

[18] B. Kilic, U. Dogan, A. H. Parlak et al., "Ocular findings in patients with psoriasis," International Journal of Dermatology, vol. 52, no. 5, pp. 554-559, 2013.

[19] S. Omar and H. Helaly, "Prevalence of ocular findings in a sample of Egyptian patients with psoriasis," Indian Journal of Dermatology, Venereology and Leprology, vol. 84, no. 1, pp. 34-38, 2018.

[20] M. A. Radtke, I. Schäfer, G. Glaeske, A. Jacobi, and M. Augustin, "Prevalence and comorbidities in adults with psoriasis compared to atopic eczema," Journal of the European Academy of Dermatology and Venereology, vol. 31, no. 1, pp. 151-157, 2017.

[21] F. M. Türkcü, A. Şahin, H. Yüksel et al., "Evaluation of choroidal thickness in psoriasis using optical coherence tomography," International Ophthalmology, vol. 36, no. 6, pp. 851854, 2016.

[22] S. L. Zagora and P. McCluskey, "Ocular manifestations of seronegative spondyloarthropathies," Current Opinion in Ophthalmology, vol. 25, no. 6, pp. 495-501, 2014.

[23] A. M. Abu El-Asrar, S. Struyf, D. Kangave et al., "Cytokine profiles in aqueous humor of patients with different clinical entities of endogenous uveitis," Clinical Immunology, vol. 139, no. 2, pp. 177-184, 2011.

[24] K. G. Ooi, G. Galatowicz, V. L. Calder, and S. L. Lightman, "Cytokines and chemokines in uveitis: is there a correlation with clinical phenotype?," Clinical Medicine \& Research, vol. 4, no. 4, pp. 294-309, 2006.

[25] R. Queiro, I. Morante, I. Cabezas, and B. Acasuso, "HLA-B27 and psoriatic disease: a modern view of an old relationship," Rheumatology, vol. 55, no. 2, pp. 221-229, 2016.

[26] D. Wakefield, W. Yates, S. Amjadi, and P. McCluskey, "HLAB27 anterior uveitis: immunology and immunopathology," Ocular Immunology and Inflammation, vol. 24, no. 4, pp. 450-459, 2016.

[27] D. Wendling and C. Prati, "Paradoxical effects of anti-TNF- $\alpha$ agents in inflammatory diseases," Expert Review of Clinical Immunology, vol. 10, no. 1, pp. 159-169, 2013.

[28] J. Sheppard, A. Joshi, K. A. Betts et al., "Effect of adalimumab on visual functioning in patients with noninfectious intermediate uveitis, posterior uveitis, and panuveitis in the VISUAL-1 and VISUAL-2 trials," JAMA Ophthalmology, vol. 135 , no. 6 , pp. 511-518, 2017. 